\title{
Low-Dose Lipopolysaccharide Selectively Sensitizes Hypoxic Ischemia-Induced White Matter Injury in the Immature Brain
}

\author{
LAN-WAN WANG, YING-CHAO CHANG, CHANG-YI LIN, JAU-SHYONG HONG, AND CHAO-CHING HUANG \\ Institutes of Clinical Medicine [L.W.W., C.C.H.] and Basic Medical Sciences [C.Y.L.], and Department of Pediatrics [C.C.H.], National \\ Cheng Kung University College of Medicine; Department of Pediatrics [L.W.W.], Chi Mei Medical Center, Tainan 704, Taiwan; \\ Department of Pediatrics [Y.C.C.], Chang Gung Memorial Hospital-Kaohsiung Medical Center, Kaohsiung 833, Taiwan; \\ Neuropharmacology Section [J.S.H.], Laboratory of Pharmacology NIEHS/NIH, Research Triangle Park, North Carolina 27709
}

\begin{abstract}
Little is known about roles of inflammation and hypoxic ischemia (HI) in the generation of neuroinflammation and damage of blood-brain barrier (BBB) in the white matter (WM) that displays regional vulnerability in preterm infants. We investigated whether low-dose lipopolysaccharide (LPS) sensitizes HI-induced WM injury in postpartum $(\mathrm{P})$ day 2 rat pups by selectively increasing neuroinflammation and BBB damage in the WM. Pups received LPS $(0.05 \mathrm{mg} / \mathrm{kg})(\mathrm{LPS}+\mathrm{HI})$ or normal saline $(\mathrm{NS}+\mathrm{HI})$ followed by 90-min HI. LPS and NS group were the pups that had LPS or NS only. Myelin basic protein immunohistochemistry on P11 showed WM injury in LPS + HI group, but not in NS + HI, LPS, and NS groups. In contrast, no gray matter injury was found in the four groups. LPS + HI group also showed decreased number of oligodendrocytes in the WM 72-h postinsult. In the same brain region, increases of activated microglia, TNF- $\alpha$ expression, BBB leakage, and cleaved caspase-3 positive cells were much more prominent in LPS + HI group than in the other three groups 24-h postinsult. The oligodendrocytes were the major cells with cleaved caspase- 3 expression. We concluded that low-dose LPS sensitized HI-induced WM injury in the immature brain by selectively up-regulating neuroinflammation and BBB damage in the WM. (Pediatr Res 68: 41-47, 2010)
\end{abstract}

$\mathrm{D}$ espite the increase in the survival of very low birth weight (VLBW) preterm infants in recent years, cerebral palsy still occurs in $10 \%$ and cognitive/behavioral deficits in $25-50 \%$ of the very preterm survivors $(1,2)$. Periventricular white matter (WM) injury is the major brain injury and accounts for the most prominent determinant of neurologic deficits in VLBW infants (1). Epidemiologic observations show that hypoxic ischemia (HI) $(2,3)$ and inflammation (4) are the two major risk factors for WM injury or cerebral palsy in very preterm infants. Inflammation might predispose to or act in concert with $\mathrm{HI}$ in premature infants. Increased systemic cytokines in premature infants with chorioamnionitis were associated with hemodynamic disturbance leading to cerebral HI $(3,5)$, and concurrent chorioamnionitis and placental perfusion defect placed preterm infants at a higher risk of abnormal neurologic outcomes than either insult alone (6). Al-

Received September 29, 2009; accepted March 16, 2010.

Correspondence: Chao-Ching Huang, M.D., Department of Pediatrics, National Cheng Kung University Hospital, 138 Sheng-Li Road, Tainan 70428, Taiwan; e-mail: huangped@mail.ncku.edu.tw

Supported by grants from the Taiwan National Health Research Institute (NHRI-EX 97-9414NI), the National Science Counsel (NSC: 97-2811-B-006-014), Chi Mei Medical Center (CMNCKU 9802), and the Center for Gene Regulation and Signal Transduction Research, National Cheng Kung University. though most $\mathrm{HI}$ and inflammatory episodes are not life threatening and are treatable clinically, the combined effects of $\mathrm{HI}$ and inflammation during the perinatal period may exert much severe damage on the immature brain.

There are very few animal models showing inflammation plus HI selectively induces WM injury in the neonatal brain. Hagberg and coworkers and others have shown that preexposure to systemic bacterial lipopolysaccharide (LPS) (0.1-0.3 $\mathrm{mg} / \mathrm{kg}$ ) sensitized neonatal brain to HI injury (duration 20-40 $\mathrm{min}$ ) in the gray matter and WM of postpartum (P) day 7 to day 8 rodent pups (7-9). Either high-dose LPS (0.3-1 mg/kg for pregnant rats) or $\mathrm{HI}$ insult (duration lasting $50 \mathrm{~min}$ for $\mathrm{P} 9$ and $4 \mathrm{~h}$ for $\mathrm{P} 2$ pups) alone could result in both gray matter and WM injury in the immature brain (10-13). Whether low-dose LPS, which mimics less severe infection, is sufficient to selectively sensitize the developing WM to HI injury remains unknown. Premyelinating oligodendrocytes are the target cells of damage during the developmental window of vulnerability for WM injury in premature infants at 23-32 wk of gestation (14). Comparing the timing of human and rodent oligodendroglial lineage progression, the predominance of premyelinating oligodendrocytes in P2 pups (equivalent to human 20-28 wk gestation) coincides with the high-risk period of WM injury in VLBW infants (15). However, very few studies have examined whether low-dose LPS selectively sensitizes HI-induced WM injury in P2 pups.

Substantial evidence has documented a common neuroinflammatory mechanism in various neurodegenerative diseases (16). Inflammatory responses and vascular factors account for the higher susceptibility of the developing WM to HI injury (1). Animal studies showed that LPS or HI increased microglial activation and up-regulated TNF- $\alpha$ expression throughout the gray and WM in the neonatal brain (17-20). Little is known about the combined effects of low-dose LPS and HI on neuroinflammation and blood-brain barrier (BBB) damage in the WM that displays regional vulnerability in preterm infants. In this study, we hypothesized that low-dose LPS selectively sensitizes HI-induced WM injury in P2 rat pups in association with increases of neuroinflammation and BBB damage in the WM.

\footnotetext{
Abbreviations: BBB, blood-brain barrier; HI, hypoxic ischemia; IOD, integrated optical density; LPS, lipopolysaccharide; MBP, myelin basic protein; NS, normal saline; VLBW, very-low-birth-weight; WM, white matter
} 


\section{MATERIALS AND METHODS}

A neonatal rat model of cerebral WM injury. This study was approved by the Animal Care Committee at National Cheng Kung University. Rat pups were kept under standard condition with a 12/12-h light/dark cycle. We injected P2 Sprague-Dawley pups with $0.05 \mathrm{mg} / \mathrm{kg}$ LPS (Escherichia coli 0111:B4; Sigma Chemical Co.-Aldrich, St. Louis, MO) or pyrogen-free normal saline (NS) (i.p.). Pups were randomly assigned to four different groups: NS (NS injected without HI), LPS (LPS injected without HI), NS + $\mathrm{HI}$ (NS injected $3 \mathrm{~h}$ before HI) and LPS $+\mathrm{HI}$ (LPS injected $3 \mathrm{~h}$ before HI). To avoid LPS-induced body temperature changes, the rat pups were returned to their dams after a LPS or NS injection, and housed in an incubator to maintain body temperature at 33 to $34^{\circ} \mathrm{C}$ before $\mathrm{HI}$. $\mathrm{HI}$ was induced by right carotid artery ligation followed by hypoxia, as previously described (21). The right common carotid artery was permanently ligated under $2.5 \%$ halothane anesthesia. After surgery, the pups were returned to an incubator for a 1-h recovery. They were then placed in airtight $500-\mathrm{mL}$ containers partially submerged in a $36^{\circ} \mathrm{C}$ water bath, and humidified $6.5 \%$ oxygen was kept at a flow rate of $3 \mathrm{~L} / \mathrm{min}$ for $90 \mathrm{~min}$. After hypoxia, pups were returned to their dam. The rats were killed for examinations in cryosections on P3 (24-h postinsult) and P5 (72-h postinsult), and in paraffin sections on P11 (9-d postinsult).

Assessment of gray matter and WM injury. The rats were killed on P11 after pentobarbital anesthesia. After the brains had been removed and postfixed in $4 \%$ paraformaldehyde at room temperatures for $48 \mathrm{~h}$, they were dehydrated through graded alcohols and embedded in paraffin, and then coronally sectioned $(10-\mu \mathrm{m}$ thick) from the genu of the corpus callosum to the end of the dorsal hippocampus. Four sections per brain, two at the level of the striatum $(0.26 \mathrm{~mm}$ and $0.92 \mathrm{~mm}$ posterior to the bregma) and another two at the levels of the dorsal hippocampus $(3.14 \mathrm{~mm}$ and $4.16 \mathrm{~mm}$ posterior to the bregma) according to a rat brain atlas (22), were selected for immunohistochemical staining.

Nissl staining for gray matter injury. Images of Nissl-stained sections were scanned and analyzed by a computerized software (ImagePro Plus 6.0; Media Cybernetics, Bethesda, MD) to calculate the percentage of area loss in the cortex, hippocampus, and striatum in the ipsilateral versus the contralateral hemisphere (23).

Myelin basic protein (MBP) staining for WM injury. For the evaluation of WM injury, paraffin-embedded sections were deparaffinized and hydrated through graded alcohols. Endogenous peroxidase was eradicated for $30 \mathrm{~min}$ in $0.3 \% \mathrm{H}_{2} \mathrm{O}_{2}$ in methanol. Heat-induced antigen retrieval was subsequently performed using $10 \mathrm{mM}$ citrate buffer $(\mathrm{pH}=6.0)$ for $10 \mathrm{~min}$ in a microwave oven. After permealization and blocking of nonspecific binding, sections were first incubated at $4^{\circ} \mathrm{C}$ overnight with the primary anti-MBP MAb (1: 100; Chemicon), rinsed, and then incubated for $1 \mathrm{~h}$ at room temperature with biotinylated goat anti-rat $\operatorname{IgG}(1: 200$; Santa Cruz). Positively stained cells were visualized using avidin-biotin-peroxidase complex amplification (Pierce Biotechnology) with diaminobenzidine tetrahydrochloride detection. MBP expression was assessed in three regions within the WM in each hemisphere of each section, and graded using a modified 4-point scoring system (24): 0, loss of processes and complete loss of capsule; 1, loss of processes with thinning or breaks in capsule; 2, complete loss of processes with intact capsule; 3 , partial loss of processes; 4, no MBP loss. The scores of each region were summed up to obtain a total score (range, $0-12$ ) for each hemisphere. Each section had a total MBP score in the ipsilateral and contralateral hemisphere, respectively. Two independent observers, blind to the treatment conditions, measured the degrees of gray matter, and WM injury.

Evaluation of oligodendrocyte progenitors. To assess the extent of damage to the oligodendrocyte progenitors, the numbers of O4-positive cells (anti-O4 IgM 1:100; Chemicon) in the WM were compared among the four groups on P5 (72-h postinsult). O4 positive cells were counted and averaged from the three visual fields in the WM of the four brain sections as described later.

Evaluation of neuroinflammation, BBB permeability and cell apoptosis. Rat pups were killed and perfused on P3 (24-h postinsult), and the brains were then postfixed in ice-cold $4 \%$ paraformaldehyde overnight, dehydrated using $30 \%$ (wt/vol) sucrose in PBS for $2 \mathrm{~d}$, and coronally sectioned $(20-\mu \mathrm{m}$ thick) from the genu of the corpus callosum to the end of the dorsal hippocampus. Four coronal sections, as described earlier, were assessed for each brain. Immunohistochemistry for microglial cell activation (ED1), TNF- $\alpha, \operatorname{IgG}$, and cleaved caspased-3 were done. IgG extravasation was used as an indicator of BBB permeability (12). The specific primary antibodies included anti-ED1 (1:100, Chemicon), polyclonal anti-TNF- $\alpha$ (1:100; Bender MedSystems), anti-IgG (HRP-conjugated 1:200; Chemicon), and cleaved caspased-3 (1:100; Cell Signaling). Biotinylated secondary antibodies included anti-mouse IgG and anti-rabbit IgG (all 1:200). Biotin-peroxidase signals were detected using
$0.5 \mathrm{mg} / \mathrm{mL} 3^{\prime} 3^{\prime}$-diaminobenzidine (DAB)/0.003\% $\mathrm{H}_{2} \mathrm{O}_{2}$ as a substrate. Results were recorded using a microscope (BX51; Olympus).

Analysis of immunohistochemical staining. Measurement of MBP scores, the number of O4, ED1 and cleaved caspase-3-positive cells, and of the integrated OD (IOD) of TNF- $\alpha$ and IgG signals were respectively analyzed as described previously (25), using an imaging software (ImagePro Plus 6.0) at $400 \times$ magnification per visual field $\left(0.0356 \mathrm{~mm}^{2}\right)$ for $\mathrm{O} 4, \mathrm{ED} 1$ and cleaved caspase-3, and at $200 \times$ magnification per visual field $\left(0.145 \mathrm{~mm}^{2}\right)$ for MBP scores, and TNF- $\alpha$ and IgG signals. Three visual fields in the cortex and three in the subcortical WM in the medial, middle, and lateral area of each hemisphere per section (Fig. 1C), and four sections per brain as described earlier were analyzed and averaged, respectively. The mean IOD values in the WM and the cortex in the ipsilateral and contralateral hemisphere of each experimental group were compared with those of the NS group to obtain the relative IOD ratios.

Immunofluorescence. After blocking $(1 \times \mathrm{PBS}, 2 \%$ normal goat serum and $0.1 \%$ Triton $\mathrm{X}-100$ ) for $1 \mathrm{~h}$, the sections were incubated overnight at $4{ }^{\circ} \mathrm{C}$ with a mixture of two of the following primary antibodies: anti-ED1 $(1: 100)$, anti- TNF- $\alpha$ (1:100), anti-O4 IgM (1:100), anti-GFAP (1:200; Chemicon) and anti-cleaved caspase-3 (1:100). The sections were washed three times with 0.1 M PBS and then incubated with Alexa Fluor 594 anti-mouse $\mathrm{IgG} / \mathrm{IgM}$ or Alexa Fluor 488 anti-rabbit IgG (1:400; Invitrogen) for $1 \mathrm{~h}$ at room temperature. Slides were photographed for red (Alexa Fluor 594) and green (Alexa Fluor 488) fluorescence with a fluorescent microscope (E400; Nikon Instech, Kawasaki, Japan).

Statistical analysis. Statistical significance $(p<0.05)$ was determined using one-way ANOVA, and Tukey's method was used for posthoc comparisons. Continuous data are means \pm SEM.

\section{RESULTS}

Low-dose LPS selectively sensitized hypoxic ischemiainduced WM injury. We first determined gray matter and WM injury in P2 rat pups in response to different dose $(0.05,0.5$, and $1 \mathrm{mg} / \mathrm{kg}$ ) of systemic LPS injection. Neuropathological examinations performed on P11 showed that LPS-treated groups had no significant brain area loss in the cortex, striatum, and hippocampus compared with NS-treated group (Fig. $1 A$ and $B$ ). The pups treated with $1 \mathrm{mg} / \mathrm{kg}$ LPS (mortality $27 \%$ ) but not with $0.05 \mathrm{mg} / \mathrm{kg}$ (mortality $0 \%$ ) or $0.5 \mathrm{mg} / \mathrm{kg}$ LPS (mortality 27\%), had significant WM injury compared with the pups treated with NS (mortality 0\%) (Fig. $1 A$ and $D$ ). We therefore used $0.05 \mathrm{mg} / \mathrm{kg}$ LPS for the following HI experiments.

We then examined whether WM injury could be selectively induced by low-dose $(0.05 \mathrm{mg} / \mathrm{kg})$ LPS followed by $\mathrm{HI}$ in $\mathrm{P} 2$ rat pups. Nissl staining performed on P11 in the NS, LPS, NS + HI, and LPS + HI groups showed no gross gray matter injury in the four groups (Fig. 2A), and quantitative analysis also demonstrated no significant area loss in the cortex, striatum and hippocampus of the four groups (Fig. 2B). For WM injury, the LPS + HI group had significantly decreased MBP expression in the WM of the ipsilateral hemisphere than the NS, LPS and NS + HI group, whereas the four groups had similar MBP expression in the contralateral hemispheres (Fig. $2 C$ and $D$ ).

We next examined whether there were changes in the numbers of oligodendrocyte progenitors, the target cells of WM injury in preterm infants, at 72-h postinsult. Immunohistochemical studies demonstrated that the LPS + HI group had significantly decreased number of O4-positive oligodendrocyte progenitors in the WM of the ipsilateral hemisphere than the NS, LPS, and NS + HI group, whereas no significant differences were found in the WM of the contralateral hemispheres between the four groups (Fig. $3 A$ and $B$ ). 
A
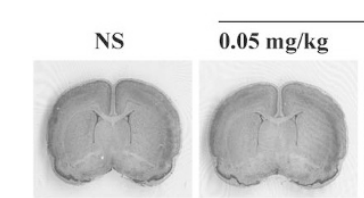

LPS

\section{A}
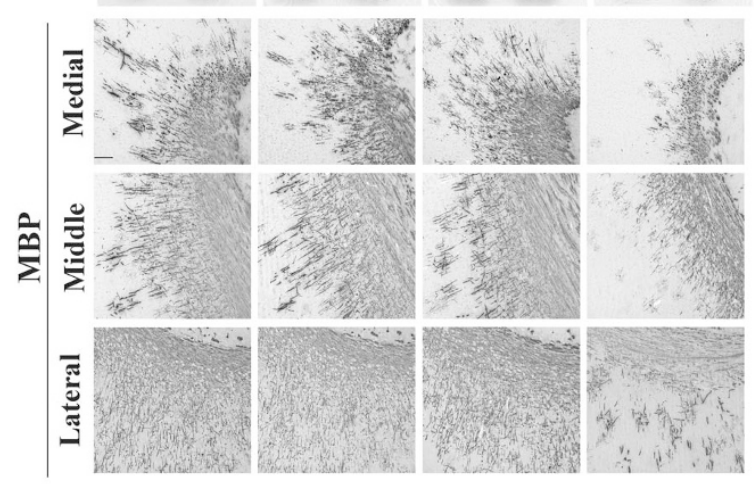

B

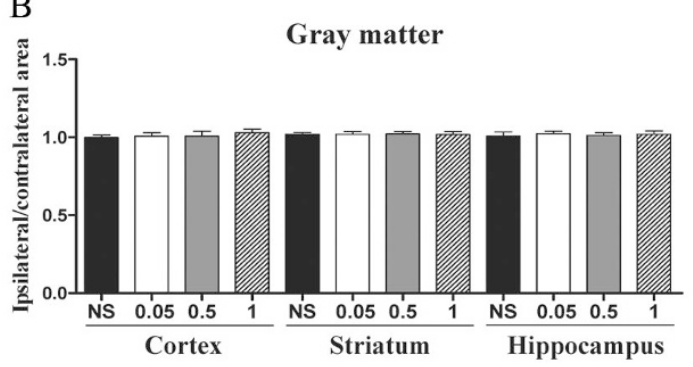

$\mathrm{C}$

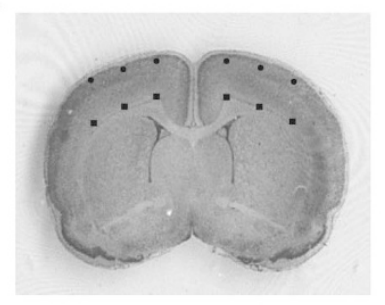

Figure 1. Nissl and MBP staining on P11 in pups treated with LPS on P2. $A$ and $B$, There was no significant area loss in the cortex, striatum and hippocampus in LPS-treated groups compared with NS-treated group. $C$, Representative section for the analysis of immunohistochemical staining in the three visual fields of the cortex (marked with circles) and three of the WM (marked with squares) in each hemisphere. $D$, Pups treated with 1 -mg $/ \mathrm{kg}$ LPS $(n=6)$, but not with $0.05 \mathrm{mg} / \mathrm{kg}$ $(n=4)$ or $0.5 \mathrm{mg} / \mathrm{kg}$ LPS $(n=6)$, had significant decreases of MBP scores compared with pups treated with NS $(n=5)$. MBP scores were measured from the three visual fields in the WM in each hemisphere marked by the black squares. Scale bar $=100 \mu \mathrm{m}$ in (A). Values are mean $\pm \operatorname{SEM}^{*} p<0.05$.

A

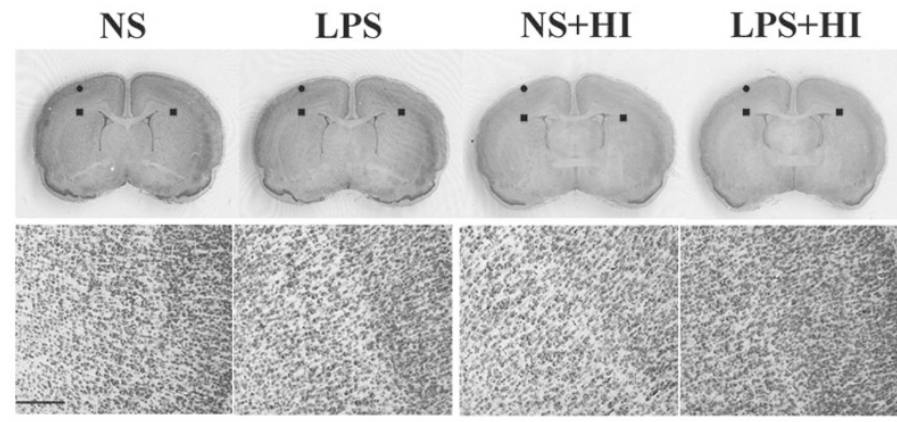

C

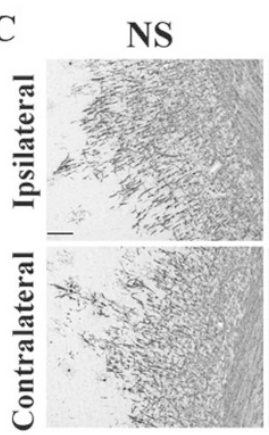

LPS

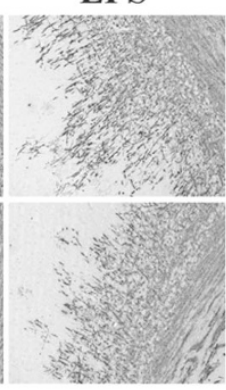

NS+HI

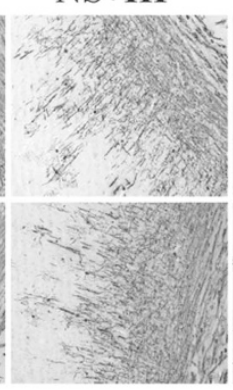

LPS+HI

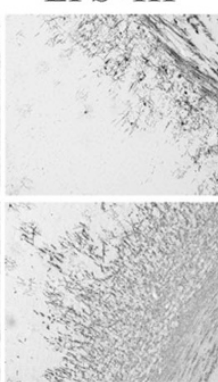

D

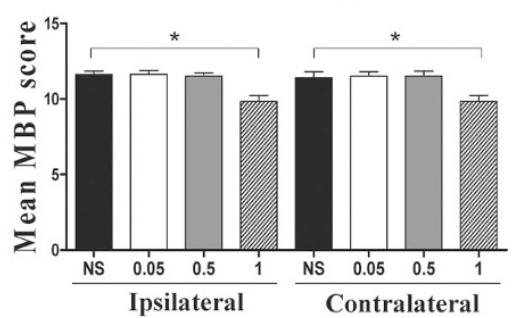

B

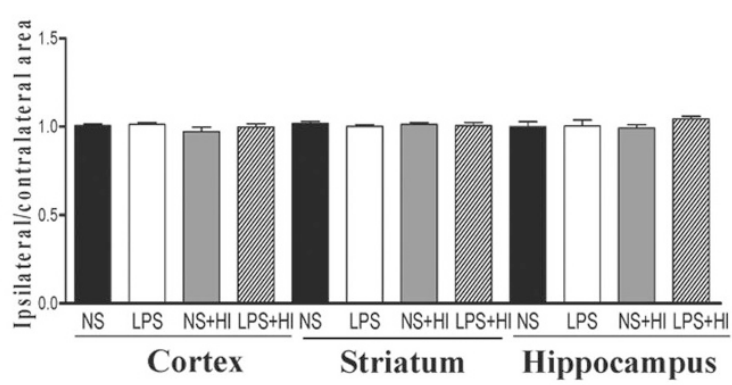

D

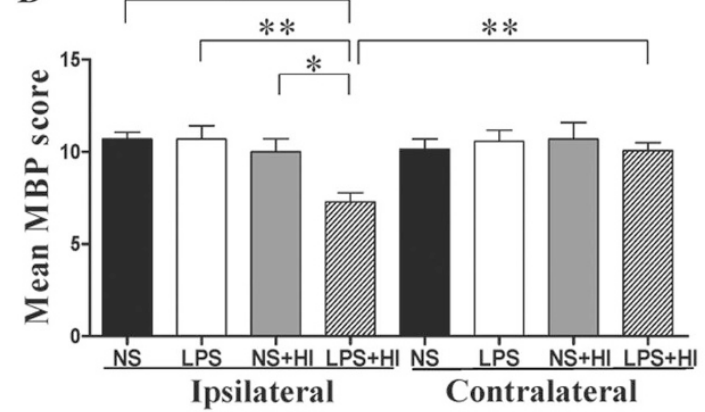

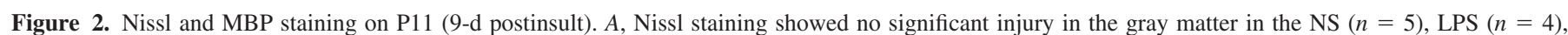

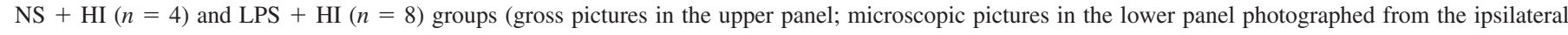

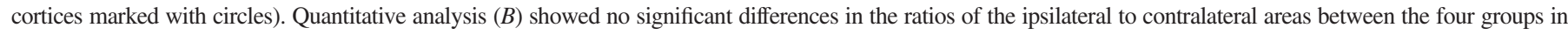

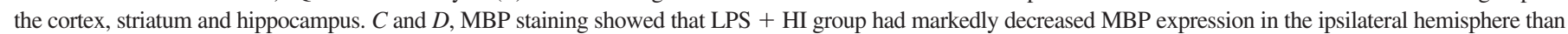

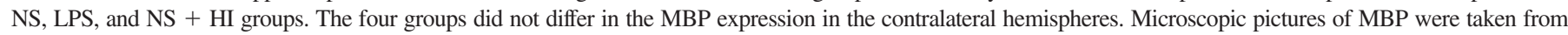
the WM area marked with squares in $(A)$. Scale bar $=200 \mu \mathrm{m}$ in $(A), 100 \mu \mathrm{m}$ in $(C)$. Values are mean $\pm \mathrm{SEM} * p<0.05 ; * * p<0.01$. 
Low-dose LPS followed by HI selectively induced neuroinflammation in the WM. At 24-h postinsult, the four groups had very few ED1-positive microglia in the bilateral cerebral cortices. In contrast, the LPS + HI group
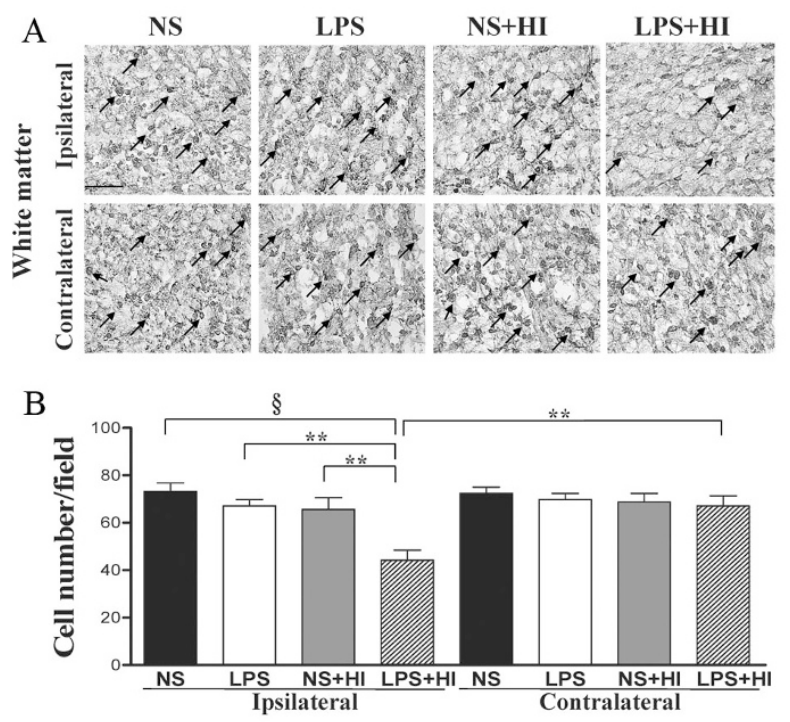

Figure 3. Immunohistochemical $(A)$ and quantitative analysis $(B)$ for $\mathrm{O} 4-$ postive oligodendrocytes (arrows) 72-h postinsult. LPS + HI group $(n=6)$ had significantly decreased numbers of O4-postive oligodendrocytes in the ipsilateral WM than NS $(n=5)$, LPS $(n=4)$, and NS + HI $(n=6)$ group. The oligodendrocyte numbers in the contralateral WM showed no difference between the four groups. Scale bar $=50 \mu \mathrm{m}$ in $(A)$. Values are means \pm SEM $* * p<0.01 ; \S p<0.001$. had significantly increased number of ED1-positive microglia in the WM of the ipsilateral hemisphere than the LPS, NS, and $\mathrm{NS}+\mathrm{HI}$ group (Fig. $4 A$ and $B$ ). The four groups had similar numbers of activated microglia in the WM of the contralateral hemispheres (data not shown).

The four groups had similar TNF- $\alpha$ expression in the bilateral cerebral cortices 24-h postinsult. In contrast, the LPS + HI group had significant increases of TNF- $\alpha$ immunoreactivities in the WM of the ipsilateral hemisphere than the NS, LPS and NS + HI group (Fig. $4 C$ and $D$ ). The TNF- $\alpha$ expression in the WM of the contralateral hemispheres did not differ between the four groups (data not shown). Immunofluorescence study in the LPS + HI group confirmed that many ED1-positive microglia colocalized with TNF- $\alpha$ (Fig. 5A).

Low-dose LPS followed by HI selectively induced BBB damage in the WM. Using IgG extravasation as a marker of BBB disruption, we found little or no $\operatorname{IgG}$ extravasation in the bilateral cerebral cortices (Fig. $6 A$ and $B$ ) or in the WM of the contralateral hemisphere of the four groups (data not shown); while the LPS + HI group had markedly increased $\mathrm{IgG}$ immunoreactivities throughout the WM in the ipsilateral hemisphere than the NS, LPS, and NS + HI group.

Low-dose LPS followed by HI caused apoptosis of oligodendrocyte progenitors in the WM. To determine the target cells of injury in response to microglial activation and BBB breakdown, cleaved caspase- 3 was used as a marker of apoptosis. The four groups had no significant differences in the numbers of cleaved caspase-3-positive cells in the bilateral cortices (Fig. $6 C$ and $D$ ), but the LPS + HI group had a

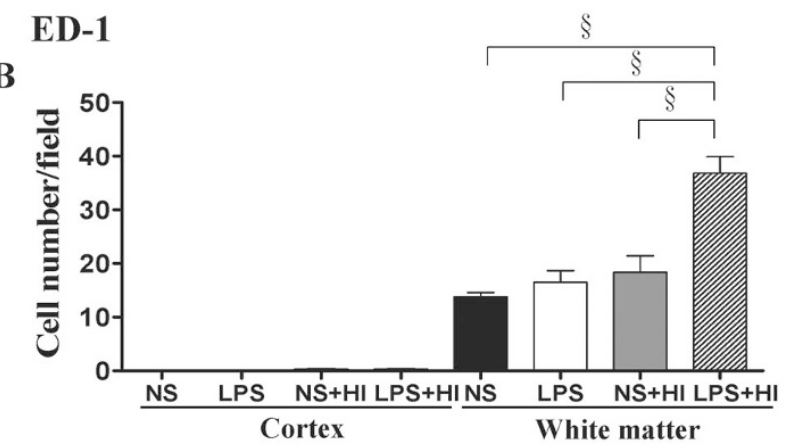

TNF- $\alpha$

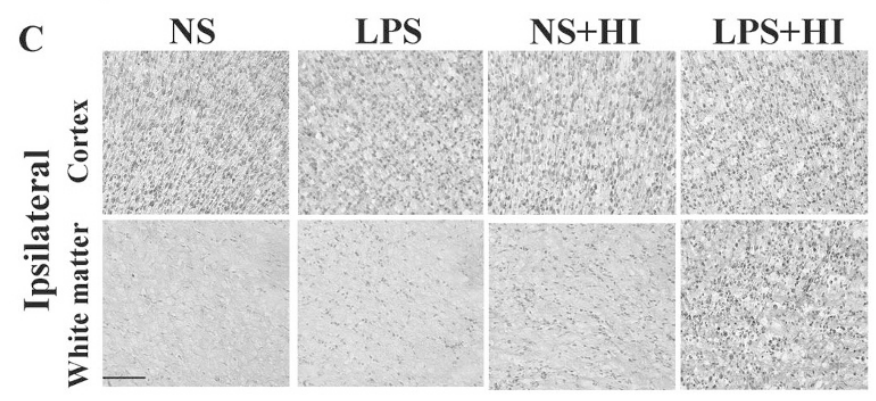

ED-1

A

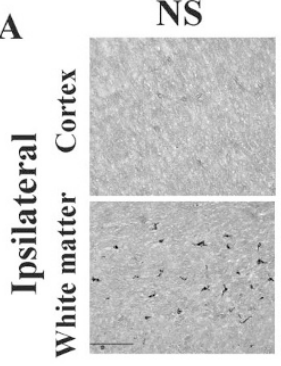

LPS
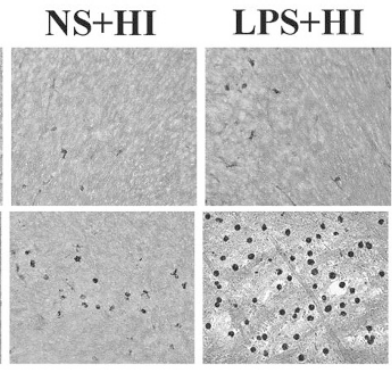

TNF- $\alpha$

D

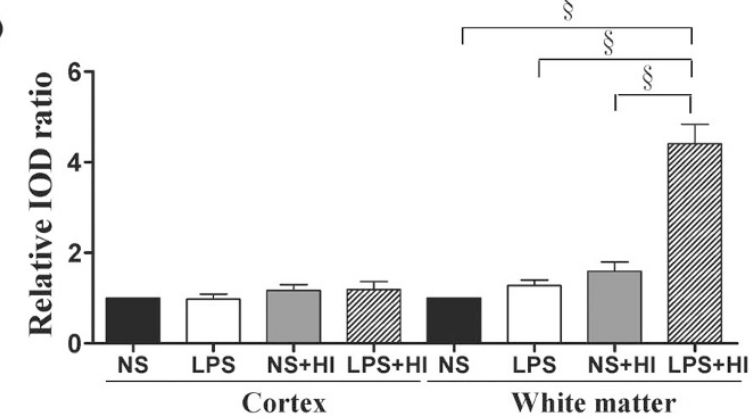

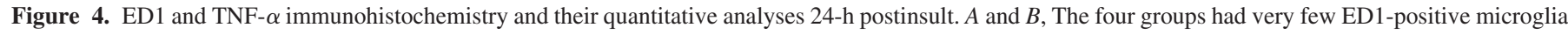

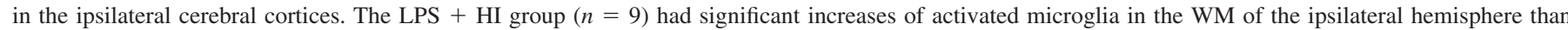

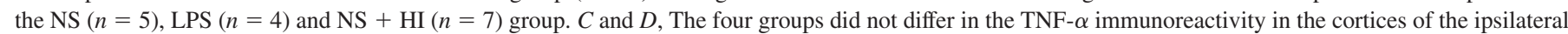

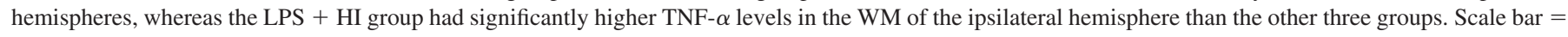
$100 \mu \mathrm{m}$ in $(A$ and $C$ ). Values are mean \pm SEM $\$ p<0.001$. 
A

B
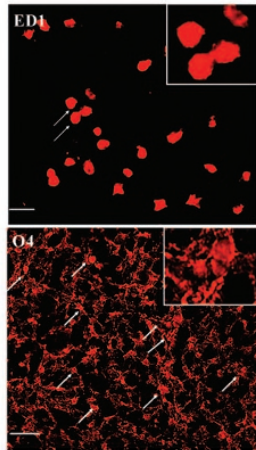

C
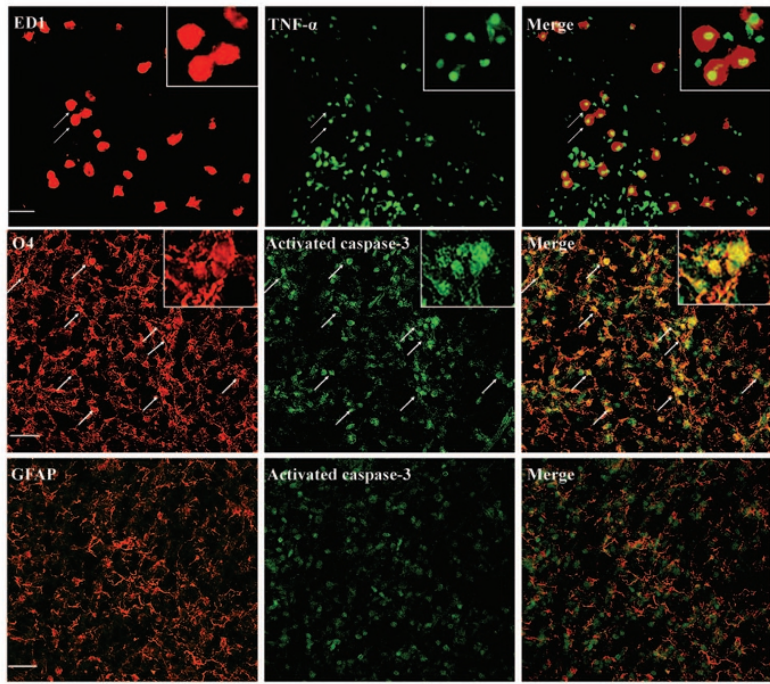

Figure 5. Immunofluorescence of the ipsilateral WM in the LPS + HI group showed that many ED1-positive microglia (arrows) colocalized with TNF- $\alpha$ $(A)$, and the cleaved caspase-3 cells were O4-postive oligodendrocytes (arrows) $(B)$ rather than GFAP-positive astrocytes $(C)$. Scale bar $=25 \mu \mathrm{m}$ in $(A-C)$.

significantly increase of cleaved caspase-3-positive cells in the WM of the ipsilateral hemisphere than the other three groups. The four groups showed no differences in the numbers of cleaved caspase-3-positive cells in the WM of the contralateral hemispheres (data not shown). The immunofluorescent pictures in the LPS + HI group showed that the O4-positive oligodendrocytes but not the GFAP- positive astrocytes, were the major cells that coexpressed cleaved caspase-3 (Fig. 5B and $C$ ).

\section{DISCUSSION}

$\mathrm{HI}$ and infection/inflammation are two major risk factors for WM injury and cerebral palsy in very preterm infants (1-4). Clinically stable preterm infants may suffer from prolonged episodes of hypoxemia without apparent apnea/bradycardia (26), and may be potentially vulnerable to impaired cerebral perfusion with small decreases in systemic blood pressure (1). The recurrent or chronic physiologic instability in VLBW infants may be associated with neurodevelopmental morbidity in early childhood (27). Sepsis occurs in $\sim 30 \%$ of very preterm infants and has shown to be associated with an increased rate of cerebral palsy (4). Culture-negative infection though may be less toxic than culture-positive sepsis, still imposes similar risk of neurodevelopmental impairment in very preterm infants as sepsis does (4). These observations raise the possibility that less severe inflammation and HI may jointly exert negative impact on the immature brain. Studies have shown that systemic LPS and HI, either given in combination or individually, induced gray matter and WM injury in neonatal rodents with age older than P7 (equivalent to $>30$ wk of human gestation) (7-13). In this study, we examined pathologic consequences on WM injury in the P2 rat brain (equivalent to VLBW infants $<30$ wk gestation) after lowdose LPS $(0.05 \mathrm{mg} / \mathrm{kg}$ ) and 90-min HI (a subthreshold HI duration for P2 pups). We demonstrated that low-dose LPS or 90-min HI alone caused no significant injury in the gray
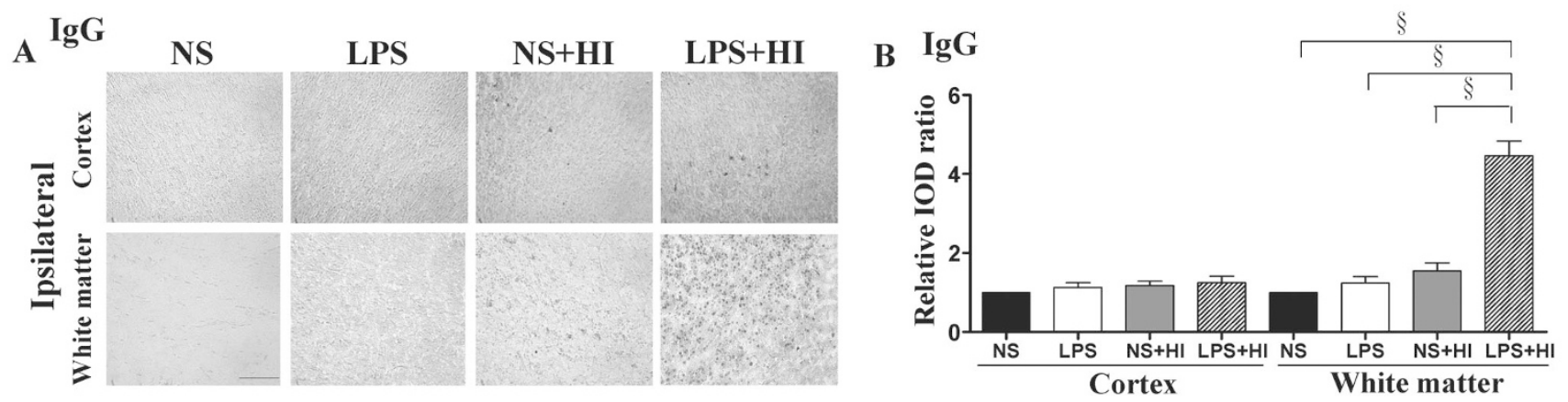

\section{Cleaved caspase-3}

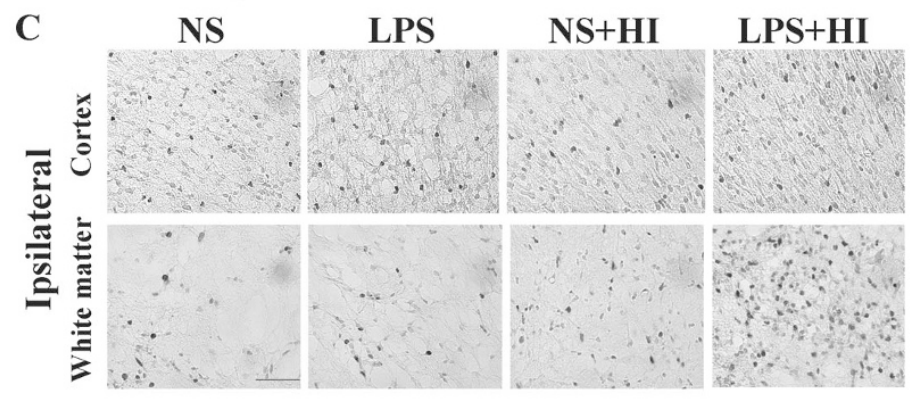

\section{Cleaved caspase- 3}

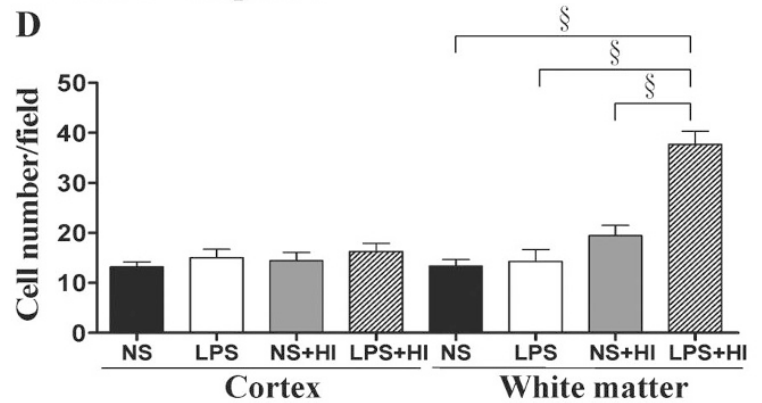

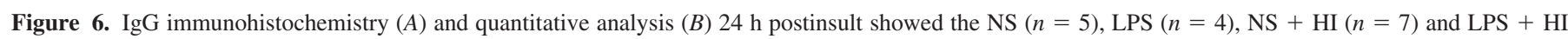

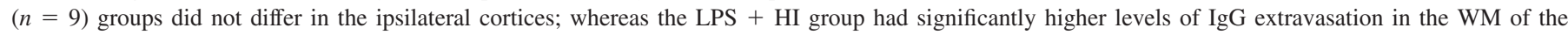

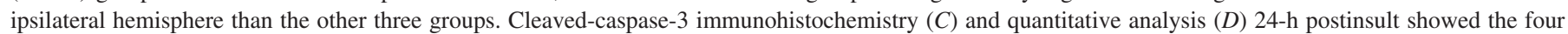

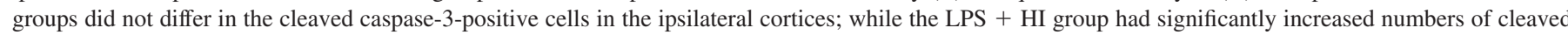

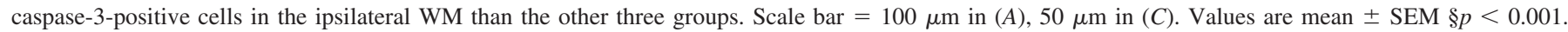


matter or WM, and that selective WM injury could only be observed by the combination of the two. WM injury induced by low-dose LPS and subthreshold HI was associated with regional increases of microglial activation, TNF- $\alpha$ expression, BBB damage, and oligodendrocyte progenitor cells apoptosis in the WM. Our study suggests that less severe inflammation and HI may cause WM injury in VLBW infants when these two events occur together.

Activated microglia are the hallmark of neuroinflammation and exacerbate brain injury through production of proinflammatory cytokines (16). Previous studies in neonatal rats showed that intracerebral LPS (10 $\mu \mathrm{g}$ for P5 pups) or HI (duration ranged from 70 min to $3 \mathrm{~h}$ for $\mathrm{P} 7$ pups) increased microglia activation (17-18) and TNF- $\alpha$ levels in the immature brain (19-20). We found that low-dose LPS or subthreshold $\mathrm{HI}$ alone did not elicit obvious microglia activation and TNF- $\alpha$ production, and also did not induce significant damage in the gray matter and WM. In contrast, low-dose LPS followed by sub-threshold $\mathrm{HI}$ increased microglia activation and TNF- $\alpha$ production selectively in the WM and caused significant WM injury. The selective increases of TNF- $\alpha$ immunoreactivities in the WM corresponded to the regionspecific activation of microglia in this P2 rat-pup model. Developmental study in fetal brains showed more activated microglia in the WM than in the overlying cortex. In addition, more pronounced activated microglia in the cerebral WM of the preterm newborn were found relative to the term newborn. The finding of a developmental-dependent abundance of activated microglia in the cerebral WM of the preterm newborns suggests a potential vulnerability of this area for brain insults characterized by activation of microglia (28).

The BBB plays an important role in brain injury induced by central- or peripheral-derived insults. In neonatal mice, moderate to severe $\mathrm{HI}$ resulted in extensive $\mathrm{BBB}$ disruption with a maximum IgG immunoreactivity at $24 \mathrm{~h}$, followed by significant gray matter and WM injury at 7-d postinsult (12). Although in neonatal rats suffering from inflammation induced by repetitive injections of LPS, increased BBB permeability was confined to the WM and associated with a substantial decrease in the WM volume (29). When both the peripheral and central insults occurred in adult mice, systemic inflammation caused sustained BBB breakdown and exacerbated ischemic brain injury (30). To extend this line of research, our study demonstrated that low-dose LPS complicated with subthreshold $\mathrm{HI}$ induced BBB disruption selectively in the WM and subsequently caused WM injury. The regional vulnerability of BBB in the WM may be related to the region-specific activation of microglia, which may contribute to BBB disruption through matrix protease generation (31). In addition, the vascularized terminal areas and the $\mathrm{BBB}$ in the periventricular WM of preterm infants are particularly susceptible to hypoperfusion due to $\mathrm{HI}$ (1). The increase of BBB permeability selectively in the WM may act in concert with microglia activation to accentuate WM injury through leukocyte recruitment into the brain (32).

Maturational vulnerability of oligodendrocytes plays another important role in the pathogenesis of WM injury. Premyelinating oligodendrocytes display greater susceptibility to oxidative damage and glutamate excitotoxicity than mature oligodendrocytes (1). In this model of low-dose LPS followed by sub-threshold HI, apoptosis occurred predominantly in the WM, and the O4-positive oligodendrocyte progenitors, mainly premyelinating oligodendrocytes in $\mathrm{P} 2$ rat brain, were the major cells that showed apoptosis. The decreased O4-positive cells corresponded to reduced MBP-expressing mature oligodendrocytes at an older age. These findings again support the maturation-dependent vulnerability to $\mathrm{WM}$ injury in $\mathrm{P} 2$ rat pups at the age equivalent to human VLBW infants (14).

Our findings are consistent with the autopsy studies in preterm infants with periventricular leukomalacia showing that activated microglia were selectively localized in the WM, and that infants with history of systemic infection and asphyxia had significant increases of TNF- $\alpha$ immunoreactivities in the WM than those with asphyxia alone (33). Our findings suggest that low-dose LPS selectively sensitizes HI-induced WM injury by regionally up-regulating neuroinflammation and BBB damage in the WM. Establishing a rat pup model of selectively acquiring WM injury through low-dose LPS and subthreshold HI may have clinical implications for further understanding the pathogenesis and development of new therapy for periventricular leukomalacia in preterm infants.

Acknowledgments. We thank Chien-Jung Ho for her skillful technical assistance with animal preparations.

\section{REFERENCES}

1. Volpe JJ 2008 Neurology of the Newborn. 5th ed, W.B. Saunders Co, Philadelphia pp 370-379, 433-436

2. Vincer MJ, Allen AC, Joseph KS, Stinson DA, Scot H, Wood E 2006 Increasing prevalence of cerebral palsy among very preterm infants: a population-based study. Pediatrics 118:e1621-e1626

3. Tsuji M, Saul JP, Plessis A, Eichenwald E, Sobh J, Crocker R, Volpe JJ 2000 Cerebral intravascular oxygenation correlates with mean arterial pressure in critically ill premature infants. Pediatrics 106:625-632

4. Stoll BJ, Hansen NI, Adams-Chapman I, Fanaroff AA, Hintz SR, Vohr B, Higgins $\mathrm{RD}$, for the National Institute of Child Health and Human development Neonatal Research Network 2004 Neurodevelopmental and growth impairment among extremely low-birth-weight infants with neonatal infection. JAMA 292:2357-2365

5. Yanowitz TD, Jordan JA, Gilmour CH, Towbin R, Bowen A, Roberts JM, Brozansk BS 2002 Hemodynamic disturbances in premature infants born after chorioamnionitis: association with cord blood cytokine concentrations. Pediatr Res 51:310-316

6. Kaukola T, Herva R, Perhomma M, Paakko E, Kingsmore S, Vainionpaa L, Hallman M 2006 Population cohort associating chorioamnionitis, cord inflammatory cytokines and neurological outcome in very preterm, extremely low birth weight infants. Pediatr Res 59:478-483

7. Lehnardt S, Massillon L, Follett P, Jensen FE, Ratan R, Rosenberg PA, Volpe JJ, Vartanian T 2003 Activation of innate immunity in the CNS triggers neurodegeneration through a Toll-like receptor 4-dependent pathway. Proc Natl Acad Sci USA 100:8514-8519

8. Eklind S, Mallard C, Leverin AL, Gilland E, Blomgren K, Mattsby-Baltzer I, Hagberg H 2001 Bacterial endotoxin sensitizes the immature brain to hypoxicischemic injury. Eur J Neurosci 13:1101-1106

9. Wang X, Svedin P, Nie C, Lapatto R, Zhu C, Gustavsson M, Sandberg M, Karlsson JO, Romero R, Hagberg H, Mallard C $2007 \mathrm{~N}$-acetylcysteine reduces lipopolysaccharide-sensitized hypoxic-ischemic brain injury. Ann Neurol 61:263-271

10. Rousset CI, Chalon S, Cantagrel S, Bodard S, Andres C, Gressens P, Saliba E 2006 Maternal exposure to LPS induces hypomyelination in the internal capsule and programmed cell death in the deep gray matter in newborn rats. Pediatr Res 59:428-433

11. Paintlia MK, Paintlia AS, Barbosa E, Singh I, Singh AK 2004 N-acetylcysteine prevents endotoxin-induced degeneration of oligodendrocyte progenitors and hypomyelination in developing rat brain. J Neurosci Res 78:347-361

12. Svedin P, Hagberg H, Savman K, Zhu C, Mallard C 2007 Matrix metalloproteinase-9 gene knock-out protects the immature brain after cerebral hypoxia-ischemia. J Neurosci 27:1511-1518

13. Back SA, Han BH, Luo NL, Chricton CA, Xanthoudakis S, Tam J, Arvin KL, Holtzman 2002 Selective vulnerability of late oligodendrocyte progenitors to hypoxia-ischemia. J Neurosci 22:455-463 
14. Back SA, Luo NL, Borenstein NS, Levin JM, Volpe JJ, Kinney HC 2001 Late oligodendrocyte progenitors coincide with the developmental window of vulnerability for human perinatal white matter injury. J Neurosci 21:1302-1312

15. Craig A, Luo NL, Beardsley DJ, Wingate-Pearse N, Walker DW, Hohimer AR, Back SA 2003 Quantitative analysis of perinatal rodent oligodendrocyte lineage progression and its correlation with human. Exp Neurol 181:231-240

16. Gao HM, Hong JS 2008 Why neurodegenerative diseases are progressive: uncontrolled inflammation drives disease progression. Trends Immunol 29:357-365

17. Fan LW, Mitchell HJ, Rhodes PG, Cai Z 2008 Alpha-phenyl-N-tert-butyl-nitrone attenuates lipopolysaccharide-induced neuronal injury in the neonatal rat brain Neuroscience 151:737-744

18. Ivacko JA, Sun R, Silverstein FS 1996 Hypoxic-ischemic brain injury induces an acute microglial reaction in perinatal rats. Pediatr Res 39:39-47

19. Fan LW, Pang Y, Lin S, Rhodes PG, Cai Z 2005 Minocycline attenuates lipopolysaccharide-induced white matter injury in the neonatal rat brain. Neuroscience 133:159-168

20. Bona E, Anderson AL, Blomgren K, Gilland E, Puka-Sundvall M, Gustafson K, Hagberg H 1999 Chemokine and inflammatory cell response to hypoxia-ischemia in immature rats. Pediatr Res 45:500-509

21. Chang YC, Huang CC, Hung PL, Huang HM 2008 Rolipram, a phosphodiesterase type IV inhibitor, exacerbates periventricular white matter lesions in rat pups. Pediatr Res 64:234-239

22. Paxinos G, Watson C 1986 The rat brain in stereotaxic coordinates. Academic Press, New York

23. Lee HT, Chang YC, Tu YF, Huang CC 2009 VEGF-A/VEGFR-2 signaling leading to cAMP response element-binding protein phosphorylation is a shared pathway underlying the protective effect of preconditioning on neurons and endothelial cells. J Neurosci 29:4356-4368
24. Manning SM, Talos DM, Zhou C, Selip DB, Park HK, Park CJ, Volpe JJ, Jensen FE 2008 NMDA receptor blockade with memantine attenuates white matter injury in a rat model of periventricular leukomalacia. J Neurosci 28:6670-6678

25. Lin HY, Huang CC, Chang KF 2009 Lipopolysaccharide preconditioning reduces neuroinflammation against hypoxic ischemia and provides long-term outcome of neuroprotection in neonatal rat. Pediatr Res 66:254-259

26. Poets CF, Stebbens VA, Richard D, Southall DP 1995 Prolonged episodes of hypoxemia in preterm infants undetected by cardiorespiratory monitors. Pediatrics 95:860-863

27. Mattia FR, deRegnier RA 1998 Chronic physiologic instability is associated with neurodevelopmental morbidity at one and two years in extremely premature infants. Pediatrics 102:e35

28. Billiards SS, Haynes RL, Folkerth RD, Trachtenberg FL, Liu LG, Volpe JJ, Kinney HC 2006 Development of microglia in the cerebral white matter of the human fetus and infant. J Comp Neurol 497:199-208

29. Stolp HB, Dziegielewska KM, Ek CJ, Potter AM, Saunders NR 2005 Long-term changes in blood-brain barrier permeability and white matter following prolonged systemic inflammation in early development in the rat. Eur J Neurosci 22:2805-2816

30. McColl BW, Rothwell NJ, Allan SM 2008 Systemic inflammation alters the kinetics of cerebrovascular tight junction disruption after experimental stroke in mice. J Neurosci 28:9451-9462

31. Zoppo GJ, Milner R, Mabuchi T, Hung S, Wang X, Berg GI, Koziol JA 2007 Microglial activation and matrix protease generation during focal cerebral ischemia. Stroke 38:646-651

32. Dammann O, Durums S, Leviton A 2001 Do white cells matter in white matter damage? Trends Neurosci 24:320-324

33. Kadhim H, Tabarki B, Verellen G, De Prez C, Rona AM, Sebire G 2001 Inflammatory cytokines in the pathogenesis of periventricular leukomalacia. Neurology $56: 1278-1284$ 